Оригинальная статья/Original article

УДК 504.7:519.7

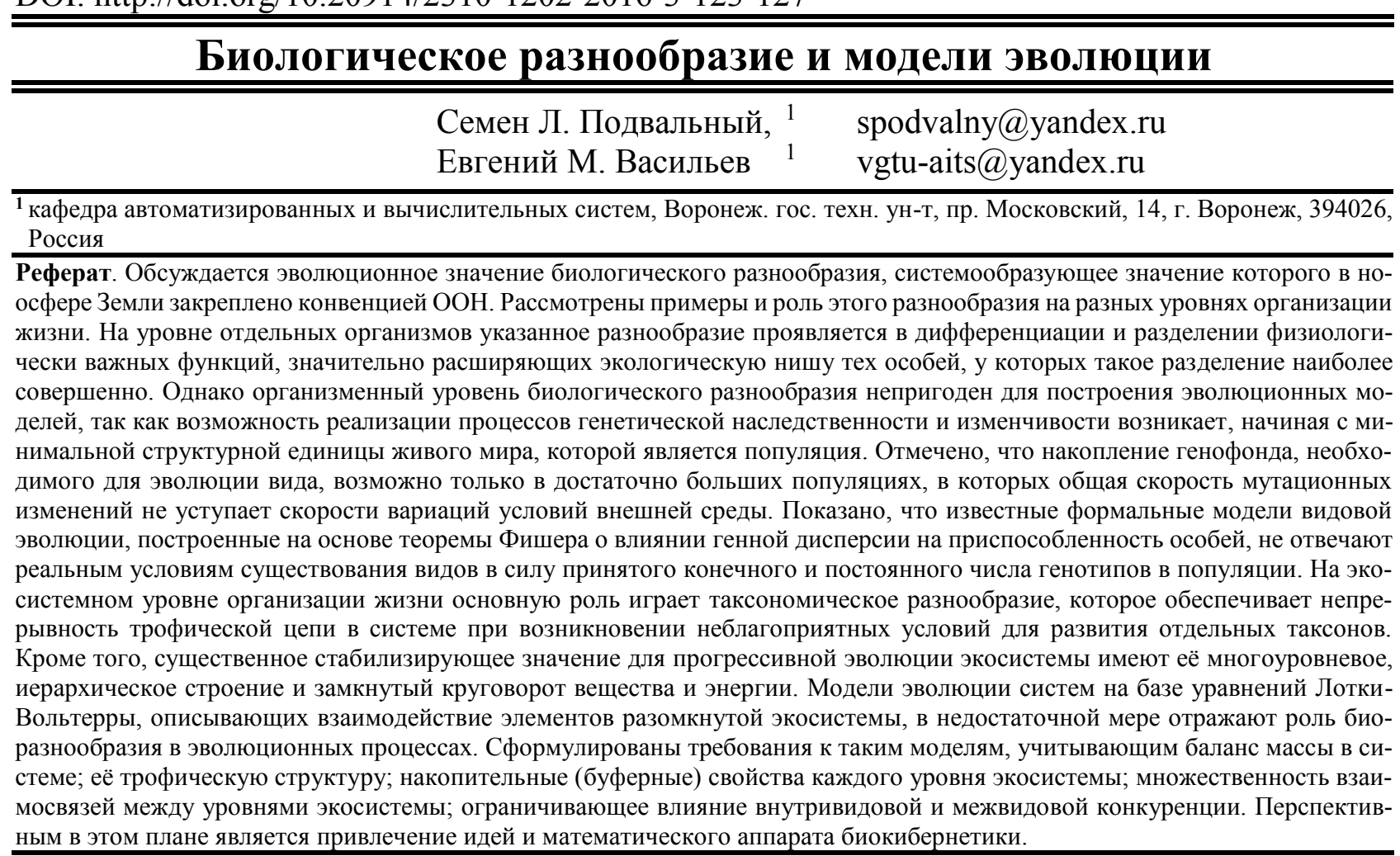

Ключевые слова: биологическое разнообразие в природе, модели эволюции

\title{
Biodiversity and models of evolution
}

Semen L. Podvalny,
Evgenii M. Vasiljev

Для цитирования

Подвальный С. Л., Васильев Е. М. Биологическое разнообразие и модели эволюции // Вестник ВГУИТ. 2016. № 3. С. 123-127. doi:10.20914/2310-1202-2016-3-123-127
For citation

Podvalny S. L., Vasiljev E. M. Biodiversity and models of evolution. Vestnik VSUET [Proceedings of VSUET]. 2016. no. 3. pp. 123-127. (in Russian). doi:10.20914/2310-1202-2016-3-123-127 


\section{Введение}

Значение биологического разнообразия, зафиксированное конвенцией ООН [1], остаётся в настоящее время широко обсуждаемым свойством природы не только в силу его стабилизирующих экосистемных функций и жизнеобеспечивающей роли для человечества, но всё в большей степени как одним из механизмов эволюционных процессов на всех уровнях организации живого мира [2-4]. Актуальность и практическая значимость понимания этого механизма определяется появившейся в настоящее время возможностью целенаправленного влияния на эволюционные процессы природы со стороны «нового геологического фактора» человека, получившего такое «определение» в работах В. И. Вернадского, раскрывшего антропогенный характер современной биосферы [5]. Научный подход к реализации указанного антропогенного воздействия на природу

Защитные жёсткие крылья и мягкие крылья для полёта

Protective hard wings and soft wings for flight

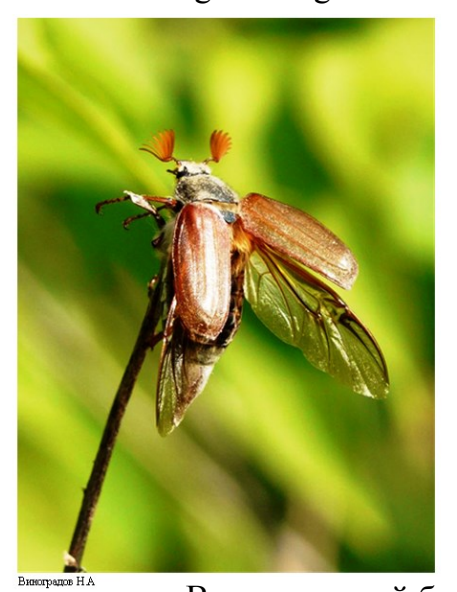

Водно-солевой баланс осетровых в реке и мор

Water-salt balance sturgeon in the river and the sea приводит к необходимости построения соответствующих формальных моделей, отражающих роль многообразия в протекании эволюционных процессов.

\section{1 Многообразие на уровне организмов}

В биологическом плане источником разнообразия живых организмов является соответствующее разнообразие их генетических структур, определяемое, в свою очередь, дискретным набором нуклеотидов, число возможных комбинаций которых в нуклеотидных последовательностях значительно превосходит количество известных в настоящее время белковых соединений.

На уровне отдельных организмов указанное разнообразие достаточно очевидно и проявляется в дифференциации и разделении физиологически важных функций, значительно расширяющих экологическую нишу тех особей, у которых такое разделение наиболее совершенно, рисунок 1.

Внутривидовое разделение на озимые и яровые формы

Intraspecific division into winter and spring forms

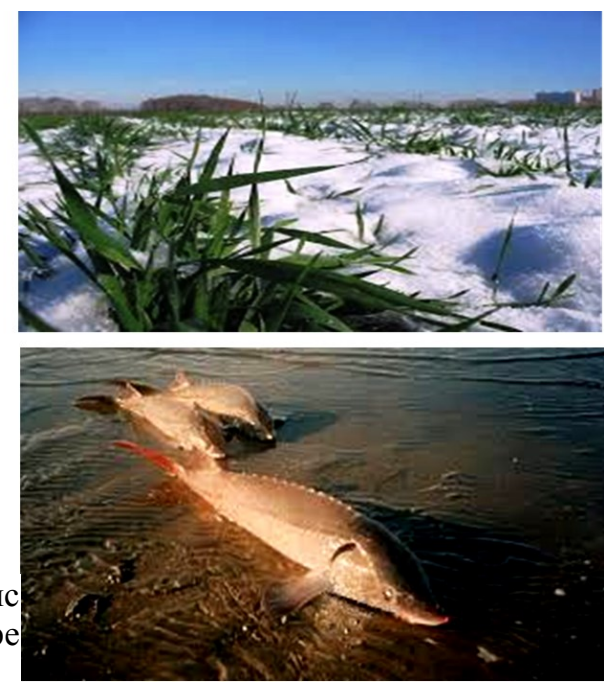

Рисунок 1. Иллюстрация примеров разделения приспособительных функций у различных биологических видов Figure 1. Illustration of examples of adaptive division of functions in different species

В качестве примеров можно привести существование у жуков Coleoptera мягких и жёстких пар крыльев, дающих жукам возможность полёта и механическую защиту при перемещениях в почве или древесине; наличие у актинобактерий рода Rhodococcus четырёх типов клеток, обеспечивающих, в результате межклеточной кооперации, жизнедеятельность бактериальных колоний в агрессивных средах с высокой степенью загрязнения минеральными солями и нефтепродуктами; существование в жаберном эпителии проходных рыб ионофильтрующих клеток как морского, так и пресноводного типов; образование раннелетних и позднелетних растений, вплоть до разделения на яровые и озимые формы одного 


\section{Becmник BTYYHST/Proceedings of VSUET, № 3, 2016}

вида. Исключительно важным фактом, подтверждающим предпочтительность биологической специализации функций перед их универсальностью, является существование у высших организмов функционально неспециализированных, так называемых стволовых клеток, способных, при возникновении необходимости в регенерации того или иного органа или ткани, дифференцироваться в специализированные клетки.

\section{2 Многообразие на уровне популяции}

Несмотря на отмеченную очевидность приведённых примеров, рассмотренный организменный уровень биологического разнообразия непригоден для построения эволюционной модели в силу того, что минимальной структурной единицей живого мира, в которой возможно протекание эволюционных процессов, является популяция. Более того, накопление генофонда, необходимого для сохранения вида, возможно только в достаточно больших популяциях, в которых общая скорость мутационных изменений не уступает скорости вариаций условий внешней среды. Указанное обстоятельство частично учитывает известная теорема Фишера [6], согласно которой средняя скорость приспособленности популяции возрастает пропорционально генной дисперсии (вариации) внутри популяции:

$$
\begin{cases}\because & \left.\sum_{j=1}^{n}\left(w_{j} x_{j}\right)\right) x_{i}\end{cases}
$$

где $w_{i}$ - степень приспособленности особей с генотипом $i ; x_{i}$ - частота встречаемости генотипа $i$ в популяции;

$$
\sum_{i=1}^{n} w_{i} x_{i}=\bar{w}
$$

где $\bar{w}$ - средняя приспособленность всех генотипов; $n$ - число различных генотипов.

Дальнейшее развитие модели Фишера связано с устранением ограничения на исходное разнообразие, накладываемое конечным числом $n$ генотипов, что позволит приблизить эту модель к реальным условиям. К таким условиям следует отнести бесконечно большое разнообразие изменений внешней среды, вследствие которых ограниченный набор генотипов в популяции постепенно будет использован и популяция будет обречена на вымирание. Отметим попутно, что это требование к модели вытекает из известного эволюционного принципа необходимого разнообразия У. Эшби: "Variety can destroy variety".

\section{3 Разнообразие на уровне экосистемы}

Переходя к биоразнообразию на экосистемном уровне организации жизни, следует, прежде всего, отметить таксономическое многообразие, обеспечивающее в экосистеме непрерывность трофической цепи в условиях, ставших неблагоприятными для развития отдельных таксонов, освободившуюся нишу которых занимают другие виды, ранее не игравшие доминирующего значения, но оказавшиеся более приспособленными к новым условиям.

Кроме таксономического разнообразия важную роль в экосистеме играет её иерархическая строение, образующее структуру с замкнутым циклом (круговоротом) вещества и энергии (рисунок 2). Наиболее известной моделью взаимодействия элементов экосистемы является балансовая модель Лотки-Вольтерры, однако аспекты эволюционной роли многообразия, иерархичности структуры экосистемы и её замкнутость в этой модели не затрагиваются. В этом плане перспективны работы в области биокибернетики $[7,8]$. Биокибернетическая модель экосистемы должна учесть следующие свойства экосистемы и особенности её функционирования:

- баланс массы - сохранение вещества и энергии, приведённой к эквивалентной массе вещества, в их замкнутом круговороте;

- трофическую структуру передачи вещества в экосистеме, для которой характерна быстро уменьшающаяся доля перерабатываемой массы вещества при переходе от нижних к верхним иерархическим уровням системы от продуцентов к консументам;

- накопительные (буферные) свойства каждого уровня экосистемы, способного создавать запас вещества, не полностью включаемого в его общий круговорот;

- многомерность (множественность) взаимосвязей каждого биологического вида любого уровня экосистемы с каждым видом нижестоящего и вышестоящего смежного уровня;

- ограничивающее влияние внутривидовой и межвидовой конкуренции каждого уровня экосистемы на скорость потребления и прироста биомассы на рассматриваемом и смежных уровнях трофической цепи. 


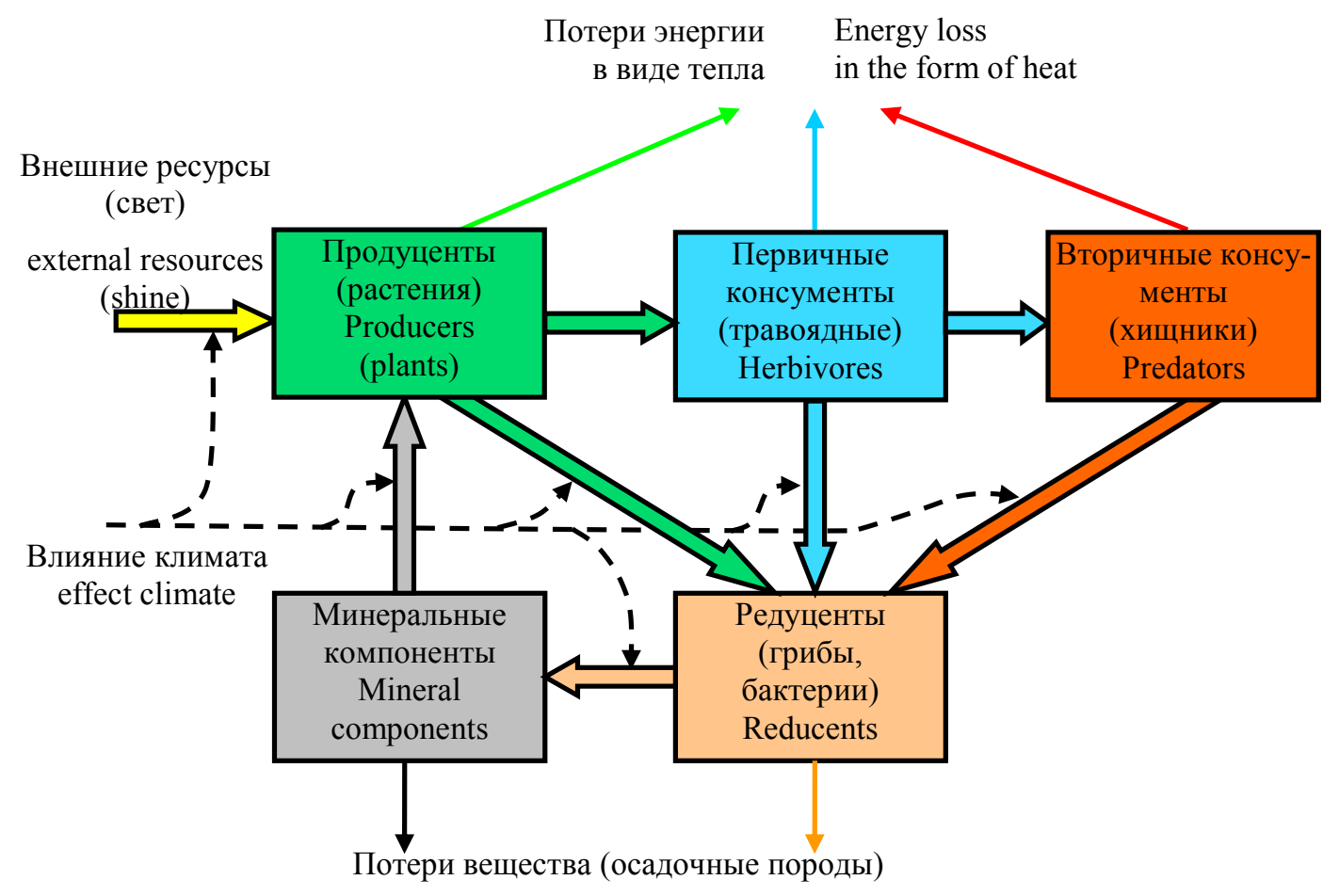

The loss of substance (sedimentary rocks)

Рисунок 2. Замкнутая структура иерархической системы

Figure 2. The closed structure of the hierarchical ecosystem

Важным аспектом построения модели функционирования экосистемы является выбор критерия её качества с точки зрения эволюционных возможностей. Традиционное моделирование экосистемы с целью анализа устойчивости не отвечает целям исследования её эволюционных свойств, так как установившееся, стабилизированное, равновесное состояние системы является эволюционно застойным. Эволюционные изменения в экосистеме представляют собою достаточно быстрый в палеонтологическом масштабе времени переходный процесс, который в простейшем случае предполагает переход из одного равновесного состояния в другое, а в более общем плане заключается в непрерывно существующем неравновесном режиме функционирования экосистемы.

\section{ЛИТЕРАТУРА}

1 Convention on biological diversity. New York: United Nations Treaty Series, 2001. V. 1760. i. nos. 30690. P. 79-307.

2 Nikisianis N., Stamou G.P. Harmony as ideology: questioning the diversity-stability hypothesis // Acta Biotheoretica. 2016. V. 64. Issue 1. P. 33-64.

3 Rodríguez R.A. et al. Thermostatistical distribution of a trophic energy proxy with analytical consequences for evolutionary ecology, species coexistence and the maximum entropy formalism // Ecological Modelling. 2014. V. 296. P. 24-35.
Биологическая сущность неравновесных режимов состоит в непрекращающейся внутри- и межвидовой конкурентной борьбе за передел существующих и освоение новых экологических ниш, причём существенные изменения окружающей среды приводят к ускоренному доминированию ранее угнетённых элементов экосистемы, обнаруживающих в новых условиях лучшие приспособительные свойства. Указанное обстоятельство было отмечено E.D. Cope [9], обратившим внимание на феноменологическое увеличение многообразия таксонов в биосистемах, оказавшихся в условиях экологического кризиса. Современный пример подобного взрывного видообразования двустворчатых моллюсков в высыхающем Аральском море подробно рассматривается в [10].

4 Venjakob C. et al. Plant diversity increases spatio-temporal niche complementarity in plant-pollinator interactions // Ecology and Evolution. 2016. V. 6. Issue 8. P. 2249-2261.

5 Вернадский В.И. Биосфера и ноосфера. М.: Айрис-пресс, 2012. 576 с.

6 Fisher R.A. The genetical theory of natural selection. Oxford: Oxford University Press, 2011. 318 p.

7 Podvalny S.L., Vasiljev E.M. A multi-alternative approach to control in open systems: origins, current state, and future prospects // Automation and Remote Control. 2015. V. 76. № 8. P. 1471-1499. 
8 Podvalny S.L., Vasiljev E.M., Barabanov V.F. Models of multi-alternative control and decisionmaking in complex system // Automation and Remote Control. 2014. V. 75. № 10. P. 1886-1891.

9 Cope E.D. The primary factors of organic evolution. Miami: HardPress Publishing, 2013.586 p.

10 Андреева С.И., Андреев Н.И. Эволюционные преобразования двустворчатых моллюсков Аральского моря в условиях экологического кризиса. Омск: Изд-во Омского гос. педагогич. ун-та, 2003. $382 \mathrm{c}$.

\section{REFERENCES}

1 Convention on biological diversity. New York, United Nations, Treaty Series, 2001, vol. 1760, i. nos. 30690, pp. 79-307.

2 Nikisianis N., Stamou G.P. Harmony as ideology: questioning the diversity-stability hypothesis. Acta Biotheoretica, 2016, vol. 64, issue 1, pp. 33-64.

3 Rodríguez R.A. et al. Thermostatistical distribution of a trophic energy proxy with analytical consequences for evolutionary ecology, species coexistence and the maximum entropy formalism. Ecological Modelling, 2014, vol. 296, pp. 24-35.

\section{СВЕДЕНИЯ ОБ АВТОРАХ}

Семён Л. Подвальный д.т.н, профессор, кафедра автоматизированных и вычислительных систем, Воронежский государственный технический университет, Московский пр-т, 14, г. Воронеж, 394026, Россия, spodvalny@yandex.ru

Евгений М. Васильев к.т.н, доцент, кафедра автоматизированных и вычислительных систем, Воронежский государственный технический университет, Московский пр-т, 14, г. Воронеж, 394026, Россия, vgtu-aits@yandex.ru

\section{КРИТЕРИЙ АВТОРСТВА}

Семён Л. Подвальный обзор литературных источников по исследуемой проблеме.

Евгений М. Васильев написал рукопись, корректировал её до подачи в редакцию и несет ответственность за плагиат.

\section{КОНФЛИКТ ИНТЕРЕСОВ}

Авторы заявляют об отсутствии конфликта интересов.

ПОСТУПИЛА 01.08.2016

ПРИНЯТА В ПЕЧАТЬ 22.08.2016
4 Venjakob C. et al. Plant diversity increases spatio-temporal niche complementarity in plant-pollinator interactions. Ecology and Evolution, 2016, vol. 6, issue 8, pp. 2249-2261.

5 Vernadskij V.I. Biosfera i noosfera [Biosphere and the noosphere]. Moscow, Ajris-press, 2012. 576 p. (in Russian).

6 Fisher R.A. The genetical theory of natural selection. Oxford, University Press, 2011. 318 p.

7 Podvalny S.L., Vasiljev E.M. A multi-alternative approach to control in open systems: origins, current state, and future prospects. Automation and Remote Control, 2015, vol. 76, no. 8, pp. 1471-1499.

8 Podvalny S.L., Vasiljev E.M., Barabanov V.F. Models of multi-alternative control and decisionmaking in complex system. Automation and Remote Control, 2014, vol. 75, no. 10, pp. 1886-1891.

9 Cope E.D. The primary factors of organic evolution. Miami, HardPress Publishing. 2013. 586 p.

10 Andreeva S.I., Andreev N.I. Evolyutsionnye preobrazovanija dvustvorchatyh molljuskov Aral'skogo morja v uslovijah jekologicheskogo krizisa [The evolutionary transformation of bivalve molluscs of the Aral Sea in the conditions of ecological crisis]. Omsk, Gos. pedagogicheskii univ., 2003. 382 p. (in Rusian.).

\section{INFORMATION ABOUT AUTHORS}

Semen L. Podvalny doc. tech. sci., professor, automation and informatics in technical systems department, Voronezh state technical university, Moscow Av., 14, Voronezh, 394026, Russia, spodvalny@yandex.ru

Evgenii M. Vasiljev cand. tech. sci., assistant professor, automation and informatics in technical systems department, Voronezh state technical university, Moscow Av., 14, Voronezh, 394026, Russia,vgtu-aits@yandex.ru

\section{CONTRIBUTION}

Semen L. Podvalny review of the literature on an investigated problem

Evgenii M. Vasiljev wrote the manuscript, correct it before filing in editing and is responsible for plagiarism

\section{CONFLICT OF INTEREST}

The authors declare no conflict of interest.

RECEIVED 8.01.2016

ACCEPTED 22.08.2016 